VoL. 73 (2006) [139-146]

\title{
SOME EXTENSIONS OF ADDITIVE PROPERTIES OF GENERAL SEQUENCES
}

\author{
Min TANG
}

Let $A=\left\{a_{1}, a_{2}, \ldots\right\}\left(a_{1}<a_{2}<\cdots\right)$ be an infinite sequence of positive integers. Let $k \geqslant 2$ be a fixed integer and denote by $R_{k}(n)$ the number of solutions of $n=a_{i_{1}}+a_{i_{2}}+\cdots+a_{i_{k}}$. Erdös, Sárközy and Sós studied the boundness of $\left|R_{2}(n+1)-R_{2}(n)\right|$ and the monotonicity property of $R_{2}(n)$. In this paper, we extend some results to $k>2$.

\section{INTRODUCTION}

Let $k \geqslant 2$ be a fixed integer and let $A=\left\{a_{1}, a_{2}, \ldots\right\}\left(a_{1}<a_{2}<\cdots\right)$ be an infinite sequence of positive integers. We write

$$
f(z)=\sum_{a \in A} z^{a}, \quad A(n)=\sum_{\substack{a \in A \\ a \leqslant n}} 1, \quad B(A, n)=\sum_{\substack{a-1 \notin A \\ a \in A, a \leqslant n}} 1 .
$$

For $n=0,1,2, \ldots$ let $R_{k}(n)$ denote the number of solutions of

$$
a_{i_{1}}+a_{i_{2}}+\cdots+a_{i_{k}}=n, \quad a_{i_{1}} \in A, a_{i_{2}} \in A, \ldots, a_{i_{k}} \in A .
$$

Then the generating function of $R_{k}(n)$ is $f^{k}(z)$.

Erdös, Sárközy and Sós studied the representation function $R_{2}(n)$. For examples, in $[2,3]$, they examined the possible order of growth of the function $R_{2}(n)$ in comparison with that of functions such as $\log n$ or $\log n \log \log n$; in [4], they showed that under certain assumptions on $A,\left|R_{2}(n+1)-R_{2}(n)\right|$ cannot be bounded; in [5], they proved that $R_{2}(n+1) \geqslant R_{2}(n)$ for all large $n$ if and only if $A(N)=N+O(1)$.

It is natural to extend these results to the case of $k$ summands, that is, to the function $R_{k}(n)$. In [6], Horváth extended the result in [2] to $k>2$. He showed that if $F(n)$ is a monotonic increasing arithmetic function with $F(n) \rightarrow+\infty$ and $F(n)=o\left(n(\log n)^{-2}\right)$, then $\left|R_{k}(n)-F(n)\right|=o\left((F(n))^{1 / 2}\right)$ cannot hold. In [1], Dombi studied the monotonicity property of $R_{k}(n)$ for $k>4$. He proved that there exists an $A \subset \mathbb{N}$ such that $R_{k}(n)$ is

Received 19th October, 2005

Supported by NNSF of China, Grant No 10471064 . The author would like to thank Professor Yong-Gao Chen for the helpful suggestions and also the referee for his/her comments and helpful suggestions.

Copyright Clearance Centre, Inc. Serial-fee code: 0004-9727/06 \$A2.00+0.00. 
increasing for every $k>4$ and $n>n_{0}(k)$ and the density of $A$ is equal to $1 / 2$. In this paper, we have the following results:

THEOREM 1. There exist infinitely many integers $N$ such that

$$
\sum_{n=0}^{N}\left(R_{k}(n+1)-R_{k}(n)\right)^{2} \geqslant c(k)(B(A, N))^{k},
$$

where $c(k)=e^{-2 k} 2^{1-2 k}(1+(2 k) !)^{-1}$.

COROLlary 1. For large enough $N$,

$$
\sum_{n=0}^{N}\left(R_{k}(n+1)-R_{k}(n)\right)^{2}=o\left((B(A, N))^{k}\right)
$$

cannot hold.

COROLLARY 2. If

$$
\lim _{N \rightarrow+\infty} \frac{B(A, N)}{N^{1 / k}}=+\infty
$$

then $\left|R_{k}(n+1)-R_{k}(n)\right|$ cannot be bounded.

TheOREM 2. If

$$
A(n)=o\left(\left(\frac{n}{\log n}\right)^{2 / k}\right),
$$

then $R_{k}(n)$ cannot be eventually monotonic increasing.

\section{Proofs}

LEMma 1. For $0<x<1$ and $m \in \mathbb{N}$, we have

$$
(1-x)^{-m-1}=1+\sum_{n=1}^{+\infty}\left(\begin{array}{c}
n+m \\
m
\end{array}\right) x^{n}>\frac{1}{m !} \sum_{n=1}^{+\infty} n^{m} x^{n} .
$$

Lemma 2. ([6]) For large $N$, we have

$$
\int_{0}^{1} \frac{1}{|1-z|} d \alpha \ll \log N
$$

where $z=e^{-1 / N} e^{2 \pi i \alpha}, \alpha$ is a real variable.

LEMMA 3. If $R_{k}(n+1) \geqslant R_{k}(n)$ for $n \geqslant n_{0}$, then

$$
R_{k}(n) \leqslant \frac{(A(2 n))^{k}}{n} \quad \text { for } n \geqslant n_{0} .
$$


Proof: For $n \geqslant n_{0}$, we have

$$
\begin{aligned}
(A(2 n))^{k} & =\left(\sum_{\substack{a \in A \\
a \leqslant 2 n}} 1\right)^{k} \geqslant \sum_{\substack{a_{i_{1}}+\cdots+a_{i_{i}} \leqslant 2 n \\
a_{i_{1}}, \ldots, a_{i_{k}} \in A}} 1=\sum_{i=1}^{2 n} R_{k}(i) \\
& \geqslant \sum_{i=n+1}^{2 n} R_{k}(i) \geqslant \sum_{i=n+1}^{2 n} R_{k}(n)=n R_{k}(n) .
\end{aligned}
$$

Hence

$$
R_{k}(n) \leqslant \frac{(A(2 n))^{k}}{n} \text { for } n \geqslant n_{0}
$$

This completes the proof of Lemma 3.

LEMMA 4. If $F(n)$ is a real arithmetic function satisfying $0 \leqslant F(n) \leqslant n$, and $F(n)=0$ holds only for finitely many integers $n$, then there exist infinitely many integers $N$ such that

$$
\frac{F(N+i)}{F(N)}<\left(\frac{N+i}{N}\right)^{2} \quad \text { for } i=1,2, \ldots
$$

Proof: Suppose that (4) holds only for finitely many $N$. Then there exists an integer $N_{0}$ such that

$$
F(N)>0 \text { for } N \geqslant N_{0} .
$$

Then there exists an integer $N^{\prime}=N^{\prime}(N)$ satisfying $N^{\prime}>N$ and

$$
\frac{F\left(N^{\prime}\right)}{F(N)} \geqslant\left(\frac{N^{\prime}}{N}\right)^{2}
$$

By induction, we get that there exist integers $N_{0}<N_{1}<N_{2}<\cdots<N_{j}<\cdots$ such that

$$
\frac{F\left(N_{j+1}\right)}{F\left(N_{j}\right)} \geqslant\left(\frac{N_{j+1}}{N_{j}}\right)^{2} \quad \text { for } j=0,1,2, \ldots
$$

Hence

$$
\begin{aligned}
F\left(N_{l+1}\right) & =F\left(N_{0}\right) \prod_{j=0}^{l} \frac{F\left(N_{j+1}\right)}{F\left(N_{j}\right)} \geqslant F\left(N_{0}\right) \prod_{j=0}^{l}\left(\frac{N_{j+1}}{N_{j}}\right)^{2} \\
& =F\left(N_{0}\right)\left(\frac{N_{l+1}}{N_{0}}\right)^{2}>N_{l+1}^{3 / 2}
\end{aligned}
$$

for large enough $l$, which contradicts the fact that $F\left(N_{l+1}\right) \leqslant N_{l+1}$.

This completes the proof of Lemma 4.

Proof of Theorem 1: If $A=\{1,2, \ldots\}$, then the result is obvious. Now let $A \subset\{1,2, \ldots\}$ be an infinite sequence and let $S(n)=\sum_{j=0}^{n}\left(R_{k}(j+1)-R_{k}(j)\right)^{2}$. Suppose 
that there are only finitely many integers $N$ satisfying (1). Then there exists an integer $N_{0}$ such that for $N \geqslant N_{0}$, we have

$$
S(N)<e^{-2 k} 2^{1-2 k}(1+(2 k) !)^{-1}(B(A, N))^{k} .
$$

By Lemma 4, there exist infinitely many integers $N$ such that

$$
\frac{B(A, N+i)}{B(A, N)}<\left(\frac{N+i}{N}\right)^{2} \quad \text { for } i=1,2, \ldots .
$$

Let $N$ denote a large integer satisfying (5) and (6). We write $e^{2 \pi i \alpha}=e(\alpha)$, and we put $r=e^{-1 / N}, z=r e(\alpha)$, where $\alpha$ is a real variable.

The infinite series

$$
f(z)=\sum_{a \in A} z^{a} \text { and } f(z)(1-z)=\sum_{n=1}^{+\infty} b_{n} z^{n}
$$

are absolutely convergent for $|z|<1$.

Let

$$
J_{1}=\int_{0}^{1}|f(z)(1-z)|^{k} d \alpha
$$

Then by Hölder's inequality and Parseval's formula,

$$
\begin{aligned}
J_{1}^{2 / k} & =\left(\int_{0}^{1}|f(z)(1-z)|^{k} d \alpha\right)^{2 / k}\left(\int_{0}^{1} 1 d \alpha\right)^{1-2 / k} \\
& \geqslant \int_{0}^{1}|f(z)(1-z)|^{2} d \alpha \\
& =\int_{0}^{1}\left|\sum_{n=1}^{+\infty} b_{n} z^{n}\right|^{2} d \alpha \\
& =\sum_{n=1}^{+\infty} b_{n}^{2} r^{2 n} \\
& \geqslant r^{2 N} \sum_{\substack{n \leqslant N \\
n \in A, n-1 \notin A}} b_{n}^{2} \\
& =e^{-2} B(A, N) .
\end{aligned}
$$

Hence

$$
J_{1} \geqslant\left(e^{-2} B(A, N)\right)^{k / 2}=e^{-k}(B(A, N))^{k / 2} .
$$

On the other hand, by Cauchy inequality and Parseval's formula, we have

$$
J_{1}=\int_{0}^{1}\left|f^{k}(z)(1-z)\right| \cdot|1-z|^{k-1} d \alpha
$$




$$
\begin{aligned}
& <2^{k-1} \int_{0}^{1}\left|f^{k}(z)(1-z)\right| d \alpha \\
& =2^{k-1} \int_{0}^{1}\left|\sum_{n=1}^{+\infty} R_{k}(n) z^{n}(1-z)\right| d \alpha \\
& =2^{k-1} \int_{0}^{1}\left|\sum_{n=1}^{+\infty}\left(R_{k}(n)-R_{k}(n-1)\right) z^{n}\right| d \alpha \\
& \leqslant 2^{k-1}\left(\int_{0}^{1}\left|\sum_{n=1}^{+\infty}\left(R_{k}(n)-R_{k}(n-1)\right) z^{n}\right|^{2} d \alpha\right)^{1 / 2} \\
& =2^{k-1}\left(\sum_{n=1}^{+\infty}\left(R_{k}(n)-R_{k}(n-1)\right)^{2} r^{2 n}\right)^{1 / 2} \\
& =2^{k-1}\left(\left(1-r^{2}\right) \frac{1}{1-r^{2}} \sum_{n=1}^{+\infty}\left(R_{k}(n)-R_{k}(n-1)\right)^{2} r^{2 n}\right)^{1 / 2} \\
& =2^{k-1}\left(\left(1-r^{2}\right) \sum_{n=1}^{+\infty} S(n-1) r^{2 n}\right)^{1 / 2} \\
& \leqslant 2^{k-1}\left(\left(1-r^{2}\right) \sum_{n=1}^{+\infty} S(n) r^{2 n}\right)^{1 / 2} \\
& =2^{k-1}\left(\left(1-e^{-2 / N}\right)\left(\sum_{n=1}^{N} S(n) r^{2 n}+\sum_{n=N+1}^{+\infty} S(n) r^{2 n}\right)\right)^{1 / 2} .
\end{aligned}
$$

For $0<x<1$, we have $1-e^{-x}<x$, and in view of (5) and (6), we have

$$
\begin{aligned}
J_{1} & <2^{k-1}\left(\frac{2}{N}\left(\sum_{n=1}^{N} S(N)+\sum_{n=N+1}^{+\infty} S(n) r^{2 n}\right)\right)^{1 / 2} \\
& =2^{k-1} \frac{1}{e^{k} 2^{k-1}(1+(2 k) !)^{1 / 2}}\left((B(A, N))^{k}+N^{-1} \sum_{n=N+1}^{+\infty}(B(A, n))^{k} r^{2 n}\right)^{1 / 2} \\
& <\frac{1}{e^{k}(1+(2 k) !)^{1 / 2}}(B(A, N))^{k / 2}\left(1+N^{-2 k-1} \sum_{n=N+1}^{+\infty} n^{2 k} r^{2 n}\right)^{1 / 2}
\end{aligned}
$$

Put $x=r^{2}$ and $m=2 k$ in Lemma 1 , and $1-e^{-x}>x / 2$ for $0<x<1$, thus

$$
\begin{aligned}
J_{1} & <\frac{1}{e^{k}(1+(2 k) !)^{1 / 2}}(B(A, N))^{k / 2}\left(1+N^{-2 k-1}(2 k) !\left(1-r^{2}\right)^{-2 k-1}\right)^{1 / 2} \\
& <\frac{1}{e^{k}(1+(2 k) !)^{1 / 2}}(B(A, N))^{k / 2}\left(1+(2 k) ! N^{-2 k-1}(1 / N)^{-2 k-1}\right)^{1 / 2} \\
& =e^{-k}(B(A, N))^{k / 2}
\end{aligned}
$$

By (7) and (8), we have

$$
e^{-k}(B(A, N))^{k / 2} \leqslant J_{1}<e^{-k}(B(A, N))^{k / 2},
$$


which is impossible, thus the assumption cannot hold.

This completes the proof of Theorem 1.

Proof of ThEOREM 2: Now suppose that (2) holds and $R_{k}(n+1) \geqslant R_{k}(n)$ for $n \geqslant n_{0}$. By Lemma 4 , there exist infinitely many integers $N$ such that

$$
\frac{A(N+i)}{A(N)}<\left(\frac{N+i}{N}\right)^{2} \quad \text { for } i=1,2, \ldots
$$

Let $N\left(\geqslant n_{0}\right)$ denote a large integer satisfying (9). We write $e^{2 \pi i \alpha}=e(\alpha)$, and we put $r=e^{-1 / N}, z=r e(\alpha)$, where $\alpha$ is a real variable. Then the infinite series $f(z)=\sum_{a \in A} z^{a}$ is
absolutely convergent for $|z|<1$.

Let

$$
J_{2}=\int_{0}^{1}|f(z)|^{k} d \alpha
$$

Then by Hölder's inequality and Parseval's formula,

$$
\begin{aligned}
J_{2}^{2 / k} & =\left(\int_{0}^{1}|f(z)|^{k} d \alpha\right)^{2 / k}\left(\int_{0}^{1} 1 d \alpha\right)^{1-2 / k} \geqslant \int_{0}^{1}|f(z)|^{2} d \alpha \\
& =\sum_{a \in A} r^{2 a} \geqslant \sum_{\substack{a \in A \\
a \leqslant N}} r^{2 N}=e^{-2} \sum_{\substack{a \in A \\
a \leqslant N}} 1=e^{-2} A(N) .
\end{aligned}
$$

Hence,

$$
J_{2} \geqslant\left(e^{-2} A(N)\right)^{k / 2}=e^{-k}(A(N))^{k / 2} .
$$

On the other hand,

$$
\begin{aligned}
J_{2} & =\int_{0}^{1}\left|f^{k}(z)\right| d \alpha \\
& =\int_{0}^{1}\left|\sum_{n=1}^{+\infty} R_{k}(n) z^{n}\right| d \alpha \\
& =\int_{0}^{1}\left|(1-z) \sum_{n=1}^{+\infty} R_{k}(n) z^{n}\right||1-z|^{-1} d \alpha
\end{aligned}
$$

Let

$$
T=\left|(1-z) \sum_{n=1}^{+\infty} R_{k}(n) z^{n}\right|
$$

Then

$$
\begin{aligned}
T & =\left|\sum_{n=1}^{+\infty}\left(R_{k}(n)-R_{k}(n-1)\right) z^{n}\right| \\
& \leqslant \sum_{n=1}^{n_{0}}\left|R_{k}(n)-R_{k}(n-1)\right| r^{n}+\sum_{n=n_{0}+1}^{+\infty}\left|R_{k}(n)-R_{k}(n-1)\right| r^{n}
\end{aligned}
$$




$$
\begin{aligned}
& <\sum_{n=1}^{n_{0}}\left|R_{k}(n)-R_{k}(n-1)\right|+\sum_{n=n_{0}+1}^{+\infty}\left(R_{k}(n)-R_{k}(n-1)\right) r^{n} \\
& <2 \sum_{n=1}^{n_{0}}\left|R_{k}(n)-R_{k}(n-1)\right|+\sum_{n=1}^{+\infty}\left(R_{k}(n)-R_{k}(n-1)\right) r^{n} \\
& =c_{1}+\sum_{n=1}^{+\infty} R_{k}(n)\left(r^{n}-r^{n+1}\right)=c_{1}+(1-r) \sum_{n=1}^{+\infty} R_{k}(n) r^{n} \\
& <c_{1}+\sum_{n=1}^{n_{0}-1} R_{k}(n)+(1-r) \sum_{n=n_{0}}^{+\infty} R_{k}(n) r^{n} \\
& <c_{2}+\left(1-e^{-1 / N}\right)\left(\sum_{n=n_{0}}^{N} R_{k}(N)+\sum_{n=N+1}^{+\infty} R_{k}(n) r^{n}\right)
\end{aligned}
$$

where $c_{1}, c_{2}$ are constants.

For $0<x<1$, we have $1-e^{-x}<x$, and in view of (3) and (9), we have

$$
\begin{aligned}
T & <c_{2}+N^{-1}\left(N \cdot \frac{(A(2 N))^{k}}{N}+\sum_{n=N+1}^{+\infty} \frac{(A(2 n))^{k}}{n} \cdot r^{n}\right) \\
& \left.<c_{2}+N^{-1}\left((A(N))^{k}(2 N / N)^{2 k}+\sum_{n=N+1}^{+\infty}(A(N))^{k}(2 n / N)^{2 k} n^{-1} r^{n}\right)\right) \\
& <c_{2}+N^{-1}(A(N))^{k} \cdot 2^{2 k}\left(1+N^{-2 k} \sum_{n=N+1}^{+\infty} n^{2 k-1} r^{n}\right) .
\end{aligned}
$$

Put $x=r$ and $m=2 k-1$ in Lemma 1 , and $1-e^{-x}(x / 2)$ for $0<x<1$, thus

$$
\begin{aligned}
T & <c_{2}+N^{-1}(A(N))^{k} \cdot 2^{2 k}\left(1+N^{-2 k} \cdot \frac{1}{(2 k-1) !}\left(1-e^{-1 / N}\right)^{-2 k}\right) \\
& <c_{2}+N^{-1}(A(N))^{k} \cdot 2^{2 k}\left(1+N^{-2 k} \frac{1}{(2 k-1) !}\left(\frac{1}{2 N}\right)^{-2 k}\right) \\
& \ll N^{-1}(A(N))^{k} .
\end{aligned}
$$

By Lemma 2, we have

$$
J_{2} \ll N^{-1}(A(N))^{k} \log N
$$

By (10) and (11), we have

$$
e^{-k}(A(N))^{k / 2} \leqslant J_{2} \ll N^{-1}(A(N))^{k} \log N .
$$

Hence

$$
(A(N))^{k / 2} \gg \frac{N}{\log N}
$$

which contradicts the assumption that $A(n)=o\left((n / \log n)^{2 / k}\right)$.

This completes the proof of Theorem 2. 


\section{REFERENCES}

[1] G. Dombi, 'Additive properties of certain sets', Acta Arith. 103 (2002), 137-146.

[2] P. Erdős and A. Sárközy, 'Problems and results on additive properties of general sequences, I', Pacific J. Math. 118 (1985), 347-357.

[3] P. Erdős and A. Sárközy, 'Problems and results on additive properties of general sequences, II', Acta Math. Hungar. 48 (1986), 201-211.

[4] P. Erdós, A. Sárközy and V.T. Sós, 'Problems and results on additive properties of general sequences, III', Studia Sci. Math. Hungar. 22 (1987), 53-63.

[5] P. Erdős, A. Sárközy and V.T. Sós, 'Problems and results on additive properties of general sequences, IV', in in: Number theory (Ootacamund, 1984), Lecture Notes in Math. 1122 (Springer-Verlag, Berlin, 1985), pp. 85-104.

[6] G. Horváth, 'On an additive property of sequences of nonnegative integers', Period. Math. Hungar. 45 (2002), 73-80.

Department of Mathematics

Anhui Normal University

Wuhu 241000

China
Department of Mathematics

Nanjing Normal University

Nanjing 210097

China

e-mail: tmzzz2000@yahoo.com 